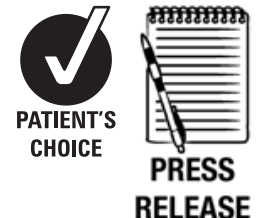

See Editorial commentary, p 593

${ }^{1}$ King's College London School of Medicine, London, UK

${ }^{2}$ Department of Clinical Neuroscience, MRC Centre for Neurodegeneration Research, King's College London, Institute of Psychiatry, London, UK ${ }^{3}$ Oxford University Nuffield Department of Clinical Neurosciences, John Radcliffe Hospital, Oxford, UK

Correspondence to Ammar Al-Chalabi, Department of Clinical Neuroscience, MRC Centre for Neurodegeneration Research, Institute of

Psychiatry, P 041, London SE5 8AF, UK;

ammar.al-chalabi@kcl.ac.uk

Received 12 January 2011 Accepted 9 March 2011

\title{
Low index-to-ring finger length ratio in sporadic ALS supports prenatally defined motor neuronal vulnerability
}

\author{
Umesh Vivekananda, ${ }^{1}$ Zita-Rose Manjalay, ${ }^{1}$ Jeban Ganesalingam, ${ }^{2}$ \\ Jacqueline Simms, ${ }^{1}$ Christopher E Shaw, ${ }^{2}$ P Nigel Leigh, ${ }^{2}$ Martin R Turner, ${ }^{3}$ \\ Ammar Al-Chalabi ${ }^{2}$
}

\begin{abstract}
Background The aetiology of apparently sporadic amyotrophic lateral sclerosis (ALS) is unknown, but prenatal factors are known to influence disease development. In both men and women, motor neurons require testosterone for survival and axonal regeneration after injury, and androgen insensitivity leads to a form of motor neuron degeneration in men. Reduction in the ratio of index to ring finger length (2D:4D ratio) is considered a surrogate marker for high prenatal testosterone levels in both men and women. The authors therefore tested the hypothesis that prenatal testosterone irrespective of gender is an independent risk factor for the development of ALS later in life, and that this would be reflected in a lower 2D:4D ratio in both men and women with ALS. Methods Patients and unrelated control individuals attending a specialist tertiary referral centre for ALS were studied. A digital camera was used to photograph hands. Finger lengths were measured by four independent scorers blind to case-control status, and the mean 2D:4D ratio derived. Analysis was by linear regression and receiver-operator-curve analysis.
\end{abstract}

Results Controlling for differences in sex ratio between groups, the 2D:4D ratio was lower for people with ALS $(n=47)$ than for controls $(n=63)(r=-0.25$, two-tailed $p=0.009$ )

Conclusions Patients with ALS have a lower 2D:4D ratio, consistent with higher prenatal circulating levels of testosterone, and possibly a prenatal influence of testosterone on motor-neuron vulnerability in later life.

\section{INTRODUCTION}

ALS is a neurodegenerative disease of upper and lower motor neurons resulting in relentlessly progressive weakness with death from respiratory failure. The median survival is 2 years from symptom onset. The aetiology of apparently sporadic ALS is unknown. Outside families with known mutations, there is currently no knowledge of the 'at risk' population for ALS, although genetic factors are known to contribute to sporadic ALS disease susceptibility, ${ }^{1}$ and athleticism has been proposed to be an environmental risk factor. ${ }^{2}$

Testosterone plays an important role in the normal function of motor neurons, which express androgen receptors. A degenerative disease of motor neurons, X linked spinobulbar muscular atrophy (Kennedy's disease), results from a trinucleotide repeat expansion in the androgen receptor gene. ${ }^{3}$ There is relative sparing of the oculomotor nuclei and Onuf's nucleus, ${ }^{4}$ which is characteristic in ALS, too. Kennedy's disease is thought to result from a combination of toxic gain of function as well as a loss of normal function of the androgen receptor. Neonatal androgen exposure in rats increases the ability of cortical neurons to respond to injury in adulthood, ${ }^{5}$ and motor neuron numbers in the spinal nucleus of the bulbocavernosus in gerbils are increased by intrauterine testosterone exposure. ${ }^{6}$ Studies of androgen insensitivity in transgenic mouse models of Kennedy's disease crossed with testicular feminisation (androgen receptor null) mice demonstrate that androgen receptor transactivation is critical for adult neuronal health and survival, and occurs in direct proportion to the sensitivity of the androgen receptor. ${ }^{7}$

Although there is no evidence for an association between alleles coding for larger androgen receptor trinucleotide repeats and ALS, ${ }^{8}$ testosterone is known to mediate motor neuron survival, dendritic length ${ }^{9}$ and axonal regeneration after injury, ${ }^{10}$ as well as being antiapoptotic, ${ }^{11}$ which are all contemporary themes in the current understanding of ALS pathophysiology, ${ }^{12}$ and it is reasonable to assume the androgen receptor is required for this protection to be mediated.

Male sex is associated with higher prenatal levels of testosterone $e^{13}$ and also with an increased risk of ALS. ${ }^{14}$ We hypothesised that a high prenatal testosterone level rather than male sex itself is an independent risk factor for the development of ALS in later life. There is evidence that the ratio of the index and ring finger lengths (2D:4D ratio) is sexually dimorphic because it is dependent on prenatal testosterone levels, with males having on average a lower ratio (relatively longer ring fingers). ${ }^{15}$ We therefore tested the hypothesis that prenatal testosterone, as an independent risk factor for the development of ALS later in life, would be reflected in a lower 2D:4D ratio for both men and women with ALS.

\section{METHODS \\ Clinical methods}

Consecutive patients and unrelated individuals attending a specialist tertiary referral centre for ALS were invited to participate. The diagnosis of ALS was made by two consultant neurologists after exclusion of other conditions. The study was ethically approved by the Joint South London and Maudsley and Institute of Psychiatry Research 
Ethics Committee, and all participants provided written informed consent prior to inclusion.

\section{Finger length measurements}

A digital camera was used to photograph hands. Photographs were taken with the hand supinated and the fingers flattened to full extension by a sheet of Perspex with the camera placed over the centre of the Perspex sheet. Digit length was measured from the basal crease of the digit to the tip using the measurement tool in Adobe Photoshop. This measurement is known to show a high degree of repeatability. ${ }^{16}$ The right hand was chosen for measurement where possible. People with faint creases and those with contractures would not be reliably measurable in this study. Rather than selecting patients for these features before entry, which might be biased and arbitrary, we invited consecutive patients to participate and then used an objective rule to exclude hands with poor measurability. Images were measured by four independent scorers (UV, JG, ZM, JS) who were blinded to case-control status. Photographs with contractures resulting in finger flexion or supination were identified by flattening and whitening of skin at the finger tips and thenar eminence by the Perspex sheet, and excluded from the analysis. Those in which the measurements made by the scorers differed by more than one SD were excluded from analysis. Remaining measurements were used and the average ratio taken as the result for that subject.

\section{Statistical methods}

The digit ratio was transformed into parametric data using a ladder of powers model and analysed with a paired $t$ test. Analyses were repeated without outliers, in case of bias introduced by extreme measurements. To confirm that the sex ratio between patient and control groups did not affect the results, we also performed linear regression with gender included as a covariate. SPSS version 15.0 was used for calculations.

\section{RESULTS}

There were 141 subjects photographed (73 patients, 68 controls). Twenty-one with contractures were excluded. The remaining 120 photographs were scored. Of these, 10 could not be measured by one of the four scorers. The remaining 110 ratios were used for analysis.

There were 47 cases (33 males, $70 \%$ ) and 63 controls (20 males, $32 \%$ ). There was no overall difference in $2 \mathrm{D}: 4 \mathrm{D}$ ratio between males and females (one-tailed $\mathrm{p}=0.41$ ).

However, the 2D:4D ratio was lower for the ALS group than controls ( $p=0.007$ ), and in linear regression with sex as a covariate this difference was independent of the sex ratio difference between the groups. The receiver-operator curve (figure 1) showed that the 2D:4D ratio was a discriminator of casecontrol status (area under the curve $=0.70, p=0.0001$ ), with a sensitivity of $72 \%$ and specificity of $65 \%$, a positive predictive value of $61 \%$ and a negative predictive value of $71 \%$.

\section{DISCUSSION}

This study has demonstrated that a group of ALS patients have a lower 2D:4D ratio in comparison with a control group, and crucially that this is independent of sex and so not explained by the higher proportion of males in the patient group. This fingerlength ratio is thought to be a marker of high prenatal testosterone levels, and our results are therefore consistent with the hypothesis that testosterone levels during development modify the subsequent risk of ALS. The digit ratio has no use as a screening tool, but the receiver-operator curve is another way

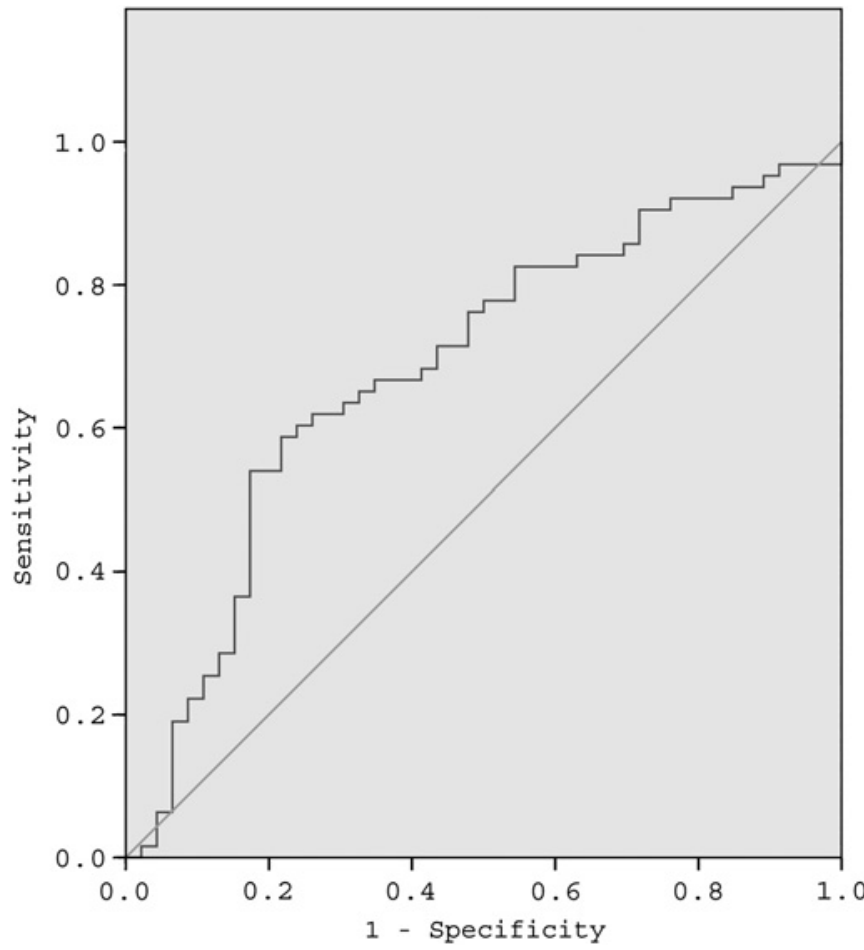

Figure 1 Receiver-operator curve for 2D:4D ratio as a predictor of amyotrophic lateral sclerosis-control status. The optimal discriminatory position in the receiver-operator curve space is the top left corner, which has $100 \%$ sensitivity and specificity. The diagonal line shows the expected plot for a random, non-discriminatory test.

of understanding the difference between the case and control 2D:4D ratios.

One limitation of this study was the exclusion of those with contractures. We attempted to limit bias by inviting every patient to participate and then analysing photographs for measurability using an objective measure of repeatability. Nevertheless, the palms with contractures are more likely to be from cases, so the analysis could not be performed in a truly blinded fashion. There is however no reason to believe that those with contractures are more likely to have higher digit ratios.

The ratio of the index and ring finger lengths (2D:4D ratio) is sexually dimorphic, ${ }^{17}$ with males having on average a lower ratio (relatively longer ring fingers). ${ }^{18}$ There was no overall effect of sex on 2D:4D ratio in this study, which might be regarded as surprising given the role of androgens in determining sexual differentiation. However, the effect of sex on 2D:4D ratio is not strong because of the large variance of the sexual dimorphism of finger lengths, and larger sample sizes than we have used are required to detect it. ${ }^{17}$

Several studies hypothesise that it is prenatal testosterone levels and sensitivity to the effects of testosterone, rather than sex, that are linked most strongly with the 2D:4D ratio, ${ }^{15} 19$ and the use of $2 \mathrm{D}: 4 \mathrm{D}$ in investigating the effects of prenatal androgens has been reviewed in comparison with other established methods. ${ }^{20}$ While direct evidence for prenatal testosterone effects on 2D:4D digit ratio is not strong, a study of 33 children who had amniocentesis and were subsequently followed supported this interpretation showing a non-significant trend towards a correlation with intrauterine testosterone and a significant association with the ratio of testosterone to oestradiol. ${ }^{21}$ Although another study did not find a relationship between second and third trimester testosterone and 2D:4D 
ratio in females, ${ }^{22}$ strong indirect evidence is that women and men with congenital adrenal hyperplasia, which leads to greatly elevated androgen levels in utero, have a low 2D:4D ratio. ${ }^{23} 24$ Thus, the $2 \mathrm{D}: 4 \mathrm{D}$ ratio is likely to be a surrogate marker for intrauterine testosterone exposure. Because high prenatal testosterone is associated with sporting ability, ${ }^{25}$ and to $2 \mathrm{D}: 4 \mathrm{D}$ ratio in women, ${ }^{16}$ it is conceivable that this is a mechanism by which the association between ALS and sporting prowess has been observed. ${ }^{26} 27$

The 2D:4D ratio does not predict adult circulating testosterone levels ${ }^{28}$ and is therefore compatible with the observation that people with ALS have low levels of free testosterone. ${ }^{29}$ The concept of intrauterine factors influencing later disease in adult life is now firmly established, ${ }^{30}$ and there is evidence that intrauterine testosterone affects neuronal function in adulthood.

High intrauterine testosterone levels might reflect pre-existing androgen insensitivity, but our reported association between low 2D:4D ratio and ALS nonetheless supports the possibility that very early factors in embryonic development might influence adult-onset motor neuronal degeneration.

Acknowledgements We thank the UK Medical Research Council and the Motor Neurone Disease Association of Great Britain and Northern Ireland for support.

Funding AA-C received support from the Myrtle Sketchley Fund.

Ethics approval Ethics approval was provided by the joint South London and Maudsley and the Institute of Psychiatry Research Ethics Committees.

Contributors The statistical analysis was carried out by UV.

Provenance and peer review Not commissioned; externally peer reviewed.

\section{REFERENCES}

1. Al-Chalabi A, Fang F, Hanby MF, et al. An estimate of amyotrophic lateral sclerosis heritability using twin data. J Neurol Neurosurg Psychiatry 2010;81:1324-6.

2. Harwood CA, McDermott CJ, Shaw PJ. Physical activity as an exogenous risk factor in motor neuron disease (MND): a review of the evidence. Amyotroph Lateral Scler 2009;10:191-204.

3. La Spada AR, Wilson EM, Lubahn DB, et al. Androgen receptor gene mutations in X-linked spinal and bulbar muscular atrophy. Nature 1991;352:77-9.

4. Sobue G, Hashizume Y, Mukai E, et al. X-linked recessive bulbospinal neuronopathy. A clinicopathological study. Brain 1989;112(Pt 1):209-32.

5. Forgie ML, Kolb B. Manipulation of gonadal hormones in neonatal rats alters the morphological response of cortical neurons to brain injury in adulthood. Behav Neurosci 2003;117:257-62

6. Forger NG, Galef BG Jr, Clark MM. Intrauterine position affects motoneuron number and muscle size in a sexually dimorphic neuromuscular system. Brain Res 1996:735:119-24.
7. Thomas PS Jr, Fraley GS, Damian V, et al. Loss of endogenous androgen receptor protein accelerates motor neuron degeneration and accentuates androgen insensitivity in a mouse model of X-linked spinal and bulbar muscular atrophy. Hum Mol Genet 2006:15:2225-38.

8. Garofalo 0, Figlewicz DA, Leigh PN, et al. Androgen receptor gene polymorphisms in amyotrophic lateral sclerosis. Neuromuscul Disord 1993:3:195-9.

9. Costa J, Gomes C, de CM. Diagnosis, pathogenesis and therapeutic targets in amyotrophic lateral sclerosis. CNS Neurol Disord Drug Targets 2010;9:764-78.

10. Perez J, Kelley DB. Trophic effects of androgen: receptor expression and the surviva of laryngeal motor neurons after axotomy. J Neurosci 1996;16:6625-33.

11. Brooks BP, Merry DE, Paulson HL, et al. A cell culture model for androgen effects in motor neurons. J Neurochem 1998;70:1054-60.

12. Rothstein JD. Current hypotheses for the underlying biology of amyotrophic latera sclerosis. Ann Neurol 2009:65(Suppl 1):S3-9.

13. Manning JT, Taylor RP. Second to fourth digit ratio and male ability in sport: implications for sexual selection in humans. Evol Hum Behav 2001:22:61-9.

14. Johnston CA, Stanton BR, Turner MR, et al. Amyotrophic lateral sclerosis in an urban setting: a population based study of inner city London. J Neurol 2006;253:1642-3.

15. Manning JT, Scutt D, Wilson J, et al. The ratio of 2nd to 4th digit length: a predictor of sperm numbers and concentrations of testosterone, luteinizing hormone and oestrogen. Hum Reprod 1998;13:3000-4.

16. Manning JT. Digit Ratio. A Pointer To Fertility, Behaviour And Health. New Jersey: Rutgers University Press, 2002

17. Baker F. Anthropological notes on the human hand. Am Anthropol 1888;1:51-76.

18. Phelps VR. Relative index finger length as a sex-influenced trait in man. Am J Hum Genet 1952:4:72-89

19. Berenbaum SA, Bryk KK, Nowak N, et al. Fingers as a marker of prenatal androgen exposure. Endocrinology 2009;150:5119-24.

20. McIntyre MH. The use of digit ratios as markers for perinatal androgen action. Reprod Biol Endocrinol 2006;4:10

21. Lutchmaya $\mathbf{S}$, Baron-Cohen $S$, Raggatt $P$, et al. 2nd to 4 th digit ratios, fetal testosterone and estradiol. Early Hum Dev 2004:77:23-8.

22. Hickey M, Doherty DA, Hart R, et al. Maternal and umbilical cord androgen concentrations do not predict digit ratio (2D:4D) in girls: a prospective cohort study. Psychoneuroendocrinology 2010;35:1235-44.

23. Okten A, Kalyoncu M, Yaris N. The ratio of second- and fourth-digit lengths and congenital adrenal hyperplasia due to 21-hydroxylase deficiency. Early Hum Dev 2002; 70:47-54.

24. Brown WM, Hines $M$, Fane BA, et al. Masculinized finger length patterns in human males and females with congenital adrenal hyperplasia. Horm Behav 2002;42:380-6.

25. Manning JT, Morris L, Caswell N. Endurance running and digit ratio (2D:4D): implications for fetal testosterone effects on running speed and vascular health. $A m J$ Hum Biol 2007;19:416-21.

26. Scarmeas N, Shih T, Stern Y, et al. Premorbid weight, body mass, and varsity athletics in ALS. Neurology 2002;59:773-5.

27. Chio A, Benzi G, Dossena M, et al. Severely increased risk of amyotrophic lateral sclerosis among Italian professional football players. Brain 2005:128:472-6.

28. Honekopp J, Bartholdt L, Beier L, et al. Second to fourth digit length ratio (2D:4D and adult sex hormone levels: new data and a meta-analytic review. Psychoneuroendocrinology 2007;32:313-21.

29. Militello A, Vitello G, Lunetta C, et al. The serum level of free testosterone is reduced in amyotrophic lateral sclerosis. J Neurol Sci 2002;195:67-70

30. Barker DJ. The fetal and infant origins of adult disease. BMJ 1990:301:1111. 